Mahendra Prasad Singh, Himanshu Roy (Hg.): Indian Political System. 3. Aufl. New Delhi: Manak Publications 2005, 546 S., € 32,40.

Harald Müller: Weltmacht Indien. Wie uns der rasante Aufstieg herausfordert. Frankfurt a. M.: Fischer Taschenbuch Verlag 2006, 350 S., € 8,95.

\section{Andreas Heinemann-Grüder}

In der bundesdeutschen universitären Politikwissenschaft, besonders im Feld der Internationalen Beziehungen und in der Vergleichenden Politikwissenschaft, fristet Indien nach wie vor ein geradezu beschämendes Schattendasein. Christian Wagners wohl informierte Einführung in das politische System Indiens könnte dem Abhilfe verschaffen, indem sie Grundkenntnisse über Institutionen, Akteure, Strukturen und Konflikte in lesbarer, knapper und übersichtlicher Form ausbreitet. Der Fokus liegt auf der institutionellen Seite des Regierungssystems; dies wird ergänzt mit einer ausgezeichneten Darstellung der Geschichte, der Funktionen und des Wandels der indischen Parteien. Unter den Politikfeldern hat sich der Autor für Themen der nationalen Integration sowie der Sozial- und Wirtschaftspolitik entschieden. Neben den Institutionen legt Wagner besonderes Augenmerk auf den indischen Politikmodus personalisierte, klientelistische Patronage-Netzwerke, Ämterkäuflichkeit, Korruption und Wahlmaschinen, die sich durch Stimmenkauf auszeichnen. Gewalt, Korruption, Einschüchterungen und Wahlfälschungen prägen laut Wagner die Wahlkämpfe (151).

Der Band beginnt mit einem Rückblick auf die präkoloniale und koloniale Erbschaft, die Unabhängigkeitsbewegung und die Kastenstruktur der indischen Gesellschaft. Im Vergleich zu den gängigen Annahmen über die Voraussetzungen einer Demokratie identifiziert der Autor mehrere „Paradoxien“: Indien habe eine Demokratie trotz des geringen Einkommensniveaus, der Defizite im Bildungsbereich, der multi-ethnischen Struktur und der zahlreichen ethnischen und religiösen Auseinandersetzungen (36 f.). Gerade die Zersplitterung, so Wagners Argument, sei eine zentrale Ursache des Zusammenhalts Indiens. Sie zwinge die Regierungen zu einer, so Wagner, „zentristischen Politik“ (37) - wobei dies auf die Unionsregierungen nach Indira Gandhi zutreffen mag, auf viele Bundesstaaten hingegen nicht. Zudem würden demokratische Verfahren auch zur Konfliktbearbeitung genutzt. Die Instabilität zahlreicher Koalitionsregierungen wiederum habe bisher keine negativen Folgen für die wirtschaftliche Entwicklung gezeitigt - eine These, die Wagner allerdings mit Blick auf die zweite Hälfte der 1990er Jahre nur eingeschränkt gelten lässt (213). Die Wirtschaftsreformen hätten trotz der Erfolge zu einem wachsenden Haushaltsdefizit, hoher Verschuldung, extremen regionalen Disparitäten und einer Konzentration von Auslandsinvestitionen in nur wenigen Bundesstaaten geführt. Während der Dienstleistungssektor boome, würden nur wenige industrielle Arbeitsplätze geschaffen, die in der Lage seien, die aus der Landwirtschaft abwandernden Gruppen aufzufangen (216).

Wagner hebt die Schwäche des Parlaments gegenüber der Exekutive hervor, insbesondere die Kompetenzarmut der zweiten Kammer; zudem beschreibt er die sukzessive Stärkung des Premierministers gegenüber dem Präsidenten und die geltenden Einschränkungen gegenüber der früher missbräuchlichen Verhängung von Not- und Ausnahmezuständen. Das Zusammenspiel von Politik, Verwaltung, Polizei und kriminellem Milieu habe, so Wagner mit Berufung auf einen früheren Vorsitzenden des Obersten Gerichts, „monströse Ausmaße“ angenommen (80).

Wagners Darstellung des Parteiensystems geht deutlich über die bekannten Einführungen in das politische System Indiens hinaus. Der Niedergang der Kongresspartei als „Regenbogenpartei", die Mobilisierung von neuen Mittelschichten durch die hindu-nationalistische BJP, die Transformation der kommunistischen Parteien und die Entstehung regionaler bzw. gruppenspezifischer Parteien (vor allem aus den Unterkasten) werden konzentriert dargestellt. Ohne Koalitionen und Sitzabsprachen mit Regionalparteien könne keine nationale Partei mehr politische Mehrheiten bilden. Die Parteien- und Wahlkampffinanzierung sei in hohem Maße klientelistisch: „Um Wähler zu gewinnen wird [...] Geld benötigt, sei es für den Stimmenkauf, für die Bereitstellung von gemeinnütziger Infrastruktur, die als Wahlgeschenk gespendet wird, oder für den parteipolitisch instrumentalisierten Missbrauch staatlicher Wohlfahrtsprogramme“ (144). 